(C) [2008] IEEE. Reprinted, with permission, from [Damith C. Herath, S. Kodagoda, and Gamini Dissanayake, New framework for Simultaneous Localization and Mapping: Multi map SLAM, Robotics and Automation, 2008. ICRA 2008. IEEE International Conference on 19-23 May 2008]. This material is posted here with permission of the IEEE. Such ermission of the IEEE does not in any way imply IEEE endorsement of any of the University of Technology, Sydney's products or services. Internal or personal use of this material is permitted. However, permission to reprint/republish this material for advertising or promotional purposes or for creating new collective works for resale or redistribution must be obtained from the IEEE by writing to pubs-permissions@ieee.org. By choosing to view this document, you agree to all provisions of the copyright laws protecting it 


\title{
New Framework for Simultaneous Localization and Mapping Multi Map SLAM
}

\author{
Damith C. Herath, S. Kodagoda, and Gamini Dissanayake
}

\begin{abstract}
The main contribution of this paper arise from the development of a new framework for the problem of Simultaneous Localization and Mapping (SLAM) in the domain of stereo vision based robot navigation. The new framework has its inspiration in the mechanics of human navigation. At present the solution is specific to a unique instance of SLAM, where the primary sensing device is a short baseline stereo vision system. The new framework addresses several key issues of this particular problem. As observed in our earlier work [1], the particular sensing device has a highly nonlinear observation model resulting in inconsistent state estimations when standard recursive estimators such as the Extended Kalman Filter (EKF) or the Unscented variants are used. Secondly, vision based approaches tend to have issues related to large feature density, narrow field of view and the potential requirement of maintaining large data bases for vision based data association techniques. The proposed Multi Map SLAM solution addresses the first issue by formulating the SLAM problem as a nonlinear batch optimization. Second issue is addressed through a two tier map representation. The two maps have unique attributes assigned to them. The Global Map (GM) is a compact global representation of the robots environment and the Local Map (LM) is exclusively used for low-level navigation between local points in the robots navigation horizon.
\end{abstract}

\section{INTRODUCTION}

$\mathrm{T}$ HIS work follows our previous work [1-3] on the use of small baseline stereo cameras in Simultaneous Localization and Mapping (SLAM). There a detailed study on sensor behavior and sensor modeling were carried out. It was shown that with the use of small baseline stereo cameras, the non linearity of the observation model manifest within very short ranges leading to inconsistencies in filter estimates. We also showed that a simple linearization as in EKF can not handle such nonlinearities requiring a more elegant solution. Here we propose nonlinear batch optimization as a suitable alternative to the standard recursive methods [4-6] used in solving the SLAM problem. We begin the batch formulation similar to $[7,8]$, however it is then extended into the new Multi Map (MM) framework where techniques from the Variable State Dimension Filter $[9,10]$ are used in order to realize a consistent and efficient

Manuscript received September 14, 2007. (Write the date on which you submitted your paper for review.) This work is supported by the ARC Centre of Excellence program, funded by the Australian Research Council (ARC) and the New South Wales State Government.

D. C. Herath, S. Kodagoda and G. Dissanayake are with the ARC Centre of Excellence for Autonomous Systems, University of Technology Sydney, AUSTRALIA (e-mail: d.herath, s.kodagoda, g.dissanayake@cas.edu.au) solution to the small base line stereo vision based SLAM problem.

The technique has evolved through observations made on human navigation patterns. It has been observed that humans tend to use a few important visual cues such as prominent constructions, major roundabouts, etc when navigating from one point of interest to the next and tend to discard most of the information utilized in refining the navigation task such as lane markings, traffic signs, etc in between such points along the path.

We intend to use a similar principle in the development of the novel Multi-Map approach. The new representation consists of a Global Map (GM), analogous to important visual cues. This map enables the SLAM algorithm to be bounded globally and the corresponding map size grows monotonically in dimensionality as the robots exploration horizon expands. The second map called the Local Map (LM) enables the lower level navigation between two visual cues of the global map, analogous to the lane markings, etc. When a particular segment of the navigation is completed, the local map corresponding to the path segment is marginalized from the robots state vector. Hence, the dimensionality of the local map state vector varies from segment to segment but does not correlate with the growth of the robots exploration horizon, considerably reducing the computational requirements of the overall SLAM algorithm. This novel representation has the added advantage of corroborating estimator consistency through explicit use of batch optimization techniques in the estimation process.

\section{SLAM as AN Optimization PRoblem}

SLAM could be posed as a multivariate parameter estimation problem $[7,8]$ where a set of unknown state variables corresponding to the robot pose and map (x) are estimated via the observations $(\mathbf{z})$ made of the environment through sensors on-board the robot. A general solution to the problem is to obtain the maximum a posteriori $(M A P)$ estimate,

$$
\begin{aligned}
& \mathbf{x}^{*} \triangleq \underset{\mathbf{x}}{\operatorname{argmax}} \quad p(\mathbf{x} \mid \mathbf{z}) \\
& =\underset{\mathbf{X}}{\operatorname{argmax}} \quad p(\mathbf{z} \mid \mathbf{x}) p(\mathbf{x})
\end{aligned}
$$

where $p(\mathbf{z} \mid \mathbf{x})$ is the observation likelihood and $p(\mathbf{x})$ is the prior. Nonlinear batch optimization techniques generally preserve the entire history of the states to be estimated. The 
large number of observations used in a single optimization cycle could potentially improve the linearization due to more accurate estimates being available for use in the linearization process.

The formulation begins by noticing that the observation likelihood in the context of SLAM contains both observations (z) to features as well as odometry measurements (u). These two types of measurements are conditionally independent and could be factorized yielding the following optimization problem,

$$
\begin{aligned}
& \mathbf{x}^{*} \triangleq \underset{\mathbf{x}}{\operatorname{argmax}} \quad p(\mathbf{z} \mid \mathbf{x}) p(\mathbf{x}) \\
& =\underset{\mathbf{x}}{\operatorname{argmax}} p(\mathbf{z}, \mathbf{u} \mid \mathbf{x}) p(\mathbf{x})
\end{aligned}
$$

Therefore, the constrained observation model is,

$$
p\left(\mathbf{z}_{i}, \mathbf{u}_{i} \mid \mathbf{x}\right)=p\left(\mathbf{z}_{i} \mid \mathbf{x}\right) p\left(\mathbf{u}_{i} \mid \mathbf{x}\right)
$$

where $\mathbf{x}$ contains both map and the entire set of poses of the robot. Assuming observations to be Gaussian distributed,

$$
p\left(\mathbf{z}_{i}, \mathbf{u}_{i} \mid \mathbf{x}\right)=\eta e^{-\frac{1}{2}\left(\boldsymbol{\varepsilon}_{i}^{T} \mathbf{R}_{i}^{-1} \boldsymbol{\varepsilon}_{i}\right)} e^{-\frac{1}{2}\left(\boldsymbol{\mu}_{i}^{T} \mathbf{U}^{-1} \boldsymbol{\mu}_{i}\right)}
$$

where, $\boldsymbol{\varepsilon}_{i}=\left(\mathbf{z}_{i}-\mathbf{h}_{i}(\mathbf{x})\right) \quad i=1, \ldots, z$ is the innovation of the stereo vision observation and $\mathbf{h}_{i}(\mathbf{x})$ is the predicted observation to the feature. $\boldsymbol{\mu}_{i}=\mathbf{u}_{i}-\mathbf{g}_{i}(\mathbf{x})$, where $\mathbf{g}_{i}(\mathbf{x})$ is the predicted odometry measurement. Now, assuming a uniform prior over the state variables, the cost function $(F(\mathbf{x}))$ to minimize in order to solve the SLAM problem is derived by taking the negative of the log of the likelihood function,

$$
\begin{aligned}
F(\mathbf{x}) & =\frac{1}{2} \sum_{i=1}^{Z} \boldsymbol{\varepsilon}_{i}^{T} \mathbf{R}_{i}^{-1} \boldsymbol{\varepsilon}_{i}+\frac{1}{2} \sum_{i=1}^{n} \mu_{i}^{T} \mathbf{U}_{i}^{-1} \mu_{i} \\
& =\frac{1}{2}\left(\boldsymbol{\varepsilon}^{T} \mathbf{R}^{-1} \boldsymbol{\varepsilon}+\mu^{T} \mathbf{U}^{-1} \mu\right)
\end{aligned}
$$

Then the new MAP estimate is,

$$
\mathbf{x}^{*}=\underset{\mathbf{x}}{\operatorname{argmin}}\left(\boldsymbol{\varepsilon}^{T} \mathbf{R}^{-1} \boldsymbol{\varepsilon}+\mu^{T} \mathbf{U}^{-1} \mu\right)
$$

This represents the standard least-squares problem generally solved through Gauss-Newton iterations,

$$
\mathbf{x}_{k+1}=\mathbf{x}_{k}+\Delta \mathbf{x}_{k}
$$

In order to minimize the cost function, the algorithm starts at $k=0$ with an initial estimate of the state vector $\hat{\mathbf{x}}_{0}$, and proceeds to calculate the approximations to the derivatives. The first derivative,

$$
\nabla F(\mathbf{x})=\mathbf{b}=-\mathbf{J}_{h}^{T} \mathbf{R}^{-1} \mathcal{\varepsilon}-\mathbf{J}_{g}^{T} \mathbf{U}^{-1} \mu
$$

and the Hessian matrix,

$$
\nabla^{2} F(\mathbf{x}) \approx \mathbf{A}=\mathbf{J}_{h}^{T} \mathbf{R}^{-1} \mathbf{J}_{h}+\mathbf{J}_{g}^{T} \mathbf{U}^{-1} \mathbf{J}_{g}
$$

Then at the $k^{\text {th }}$ iteration,

$$
\mathbf{A}_{k} \Delta \mathbf{x}_{k}=\mathbf{b}_{k}
$$

and an improved estimate of the states are realized through (7). Iterative sequence continues until an acceptable minimum is reached. A good indication of a minimum is when the rate of change in residual is minimal.

\section{MULTI MAP SLAM}

From a practical perspective MM framework utilize two different techniques for image registration corresponding to the two map representation. Features corresponding to the high level global map rely on visually salient features that can be recognized using high dimensional descriptors that are scale and affine invariant. The descriptive nature of these features provides the necessary loop closure information. In this work, we have chosen SURF [11]. SURF based Global Map (GM), is sparse and the features could only be observed at certain points of interest (PoI) in the robots trajectory. The low level map is used for navigating the robot between the features of the GM, which needs a fast feature tracker. As the features need to be tracked between consecutive frames (data association), the tracker can be simple without needing complex descriptors. Although there are several number of trackers proposed in the literature, we have chosen the KLT $[12,13]$ due to its simplicity and speed.

\section{BATCH OPTIMIZATION WITH MULTIPLE MAPS}

Let's consider the simple navigation scenario depicted in Fig.1. The robot starts at the origin and completes a small loop by returning to the origin after a temporal lapse. In this particular example, a small cluster of GM features are located near the origin of the robots trajectory marked GMnode1. There is only a unique PoI at the origin of the reference frame. Once the robot completes the journey, it sees the GM features again and realizes the loop closure. Until the robot reaches the end of the loop, it uses the features from the LM for realizing bounded estimates of its states. The algorithm that extends the batch optimization to accommodate the two maps is described next.

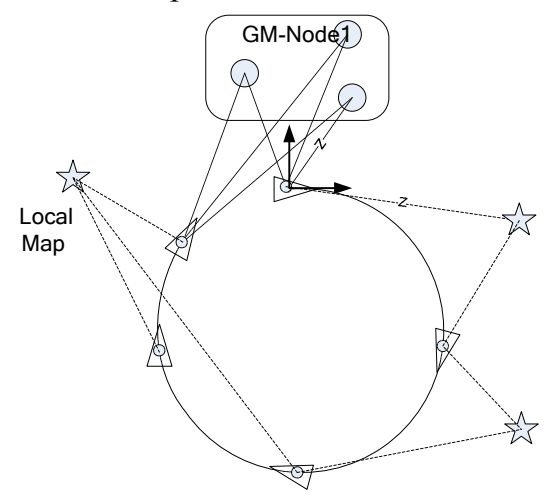

Fig. 1 Simplest form of the MM approach with a single node of Global Map. GM-Node1 provides Loop Closure information 
The first extension to the solution presented in previous section occurs when the state vector is extended to accommodate the GM features,

$$
\mathbf{x}=\left[\mathbf{x}_{r}, \mathbf{x}_{L M}, \mathbf{x}_{G M}\right]^{T}
$$

The state vector contains two independent maps. The LM ( $\mathbf{x}_{L M}$ ) contributes to the frame-to-frame optimization whilst the GM $\left(\mathbf{x}_{G M}\right)$ contributes to the loop closure. The initialization of batch optimization is provided by a local EKF, which only operate between two consecutive PoIs. After carrying out extensive simulation and experimentations, we concluded that EKF can provide a reasonable initialization for the optimization algorithm. Therefore,

$$
\hat{\mathbf{x}}_{0}=\left[\hat{\mathbf{x}}_{r}^{E K F}, \hat{\mathbf{x}}_{L M}^{E K F}, \hat{\mathbf{x}}_{G M, 0}\right]^{T}
$$

Since the Global Map features need to be integrated in the optimization algorithm, an initial estimate of the GM states $\left(\hat{\mathbf{x}}_{G M, 0}\right.$ ) is required. The GM feature observations with the EKF robot pose estimates ( $\hat{\mathbf{x}}_{r}^{E K F}$ ) is used to initialize the map. Once the state and the initial estimates are defined the minimization proceeds as discussed in the previous section.

\section{Generalized Multi MaP SMOOTHER}

In this section, we describe the generalized multi map filter for extended arbitrary navigation. Fig. 2 shows a simplified navigation scenario, which could be used to appreciate the Multi Map Smoothing (MMS) algorithm. Since the robot is not returning to its origin, the GM-node1 features can not be used in closing this larger loop causing EKF based initialization to deviate significantly. This leads to inconsistency in the estimates of the MMF. Thus, PoIs are required at regular intervals of the robot path, 1 . To maintain the consistency of the MMF estimates by executing the MMF at shorter intervals. Thereby increasing the accuracy of the initial estimates of the consecutive optimization cycles. 2. To anticipate loop closures at locations other than the origin.

We could improve the efficiency of the MMF algorithm by marginalizing LM features at appropriate intervals. Techniques based on the VSDF $[9,10]$ algorithm are used to maintain the consistency of the estimates while features are marginalized. The KLT based feature tracker is not capable of associating data with significant temporal laps. This unique character of the LM features results in marginalization exclusive of information loss.

\section{A. Estimation Process}

The algorithm could be outlined as follows. The first PoI is created at the origin (GM-Node 1 in Fig. 2) where the robot initiates its navigation as in the case of the MMF.

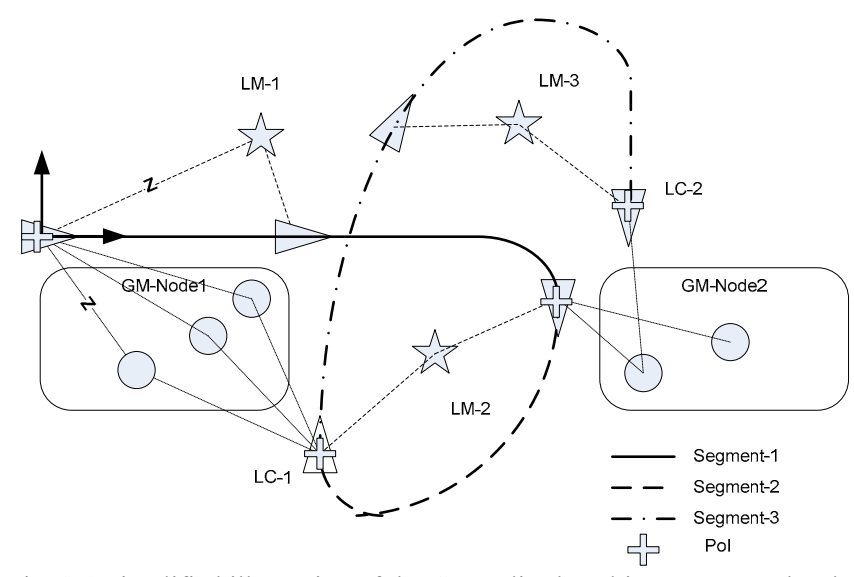

Fig. 2 A simplified illustration of the Generalised Multi Map approach. The robot starting from the origin takes a slightly complicated route. Apart from the origin, PoIs are created when the robot makes a 'sharp' turn. The first Loop Closure (LC-1) occurs when the features observed at the first PoI are observed again from the third PoI. A second Loop Closure (LC-2) occurs when some of the features observed at second PoI are re-observed from $4^{\text {th }}$ PoI.

Following PoIs are scheduled heuristically considering the anticipated navigation pattern and knowledge of the environment in which the robot operates. Thus in Fig. 2, PoIs are placed after each turn of the robot. As described previously PoIs are marked with a set of view invariant features described by high dimensional descriptors (GMnode 1,2 ) thus enabling detection of a Loop Closure. Once a PoI is reached, algorithm sets out to execute the MMF utilizing the LM and AM information currently available (for instance at LC-1 the MMF has GM-Node 1,2 and LM-2 features in the augmented map state vector). This is similar to the previously described MMF. VSDF techniques are used to marginalize the features from the state vector that do not contribute to the current execution of the MMF. The VSDF provides a means to preserve the 'contributions' to the estimator from the observations made to these marginalized features by linearizing these measurements and incorporating them into a Gaussian prior within the batch optimization paradigm.

The MMS is essentially a recursive smoother with dynamically varying state dimension. The Filter could be summarized as consisting of following components. Assuming that the robot is at the $p^{\text {th }}$ PoI, $(p>2)$ the current LM contains only a partial set of the LM features that are observed from the PoI at $p-1$. Thus the new cost function to minimize contains a prior reflecting the linearized control input data as well as the non linear measurement data,

$$
\begin{aligned}
& F(\mathbf{x})=\left(\hat{\mathbf{x}}_{0}-\mathbf{x}\right)^{T} \mathbf{A}_{\mathbf{0}}\left(\hat{\mathbf{x}}_{0}-\mathbf{x}\right)+(\mathbf{d}-f(\mathbf{x}))^{T} \mathbf{Q}^{-1}(\mathbf{d}-f(\mathbf{x})) \\
& +(\mathbf{z}-h(\mathbf{x}))^{T} \mathbf{R}^{-1}(\mathbf{z}-h(\mathbf{x}))
\end{aligned}
$$

Also it now requires augmenting the state vector with the new LM features, its corresponding observations, robot poses encompassing the current LM and any newly initialized GM features. Thus the new state change from the previous PoI, 


$$
\mathbf{x}=\left[\begin{array}{l}
\mathbf{x}_{r}(p-1) \\
\mathbf{x}_{L M}(p-1) \\
\mathbf{x}_{G M}(p-1)
\end{array}\right] \rightarrow \quad \mathbf{x}=\left[\begin{array}{l}
\mathbf{x}_{r}(p) \\
\mathbf{x}_{L M}(p) \\
\mathbf{x}_{G M}(p-1) \\
\mathbf{x}_{G M}(p)
\end{array}\right]
$$

where $\left(\mathbf{x}_{r}(p)\right)$ represents the limited pose history attributed to the $p^{\text {th }}$ LM and $\left(\mathbf{x}_{L M}(p)\right)$ contains the LM features belonging to the $p^{\text {th }}$ LM. $\mathbf{x}_{G M}(p-1)$ contains GM features that are already initialized during previous PoIs beginning from the origin and $\left(\mathbf{x}_{G M}(p)\right)$ are the newly observed GM features yet to be estimated. Thus it is clear that the only component of this dynamic state vector that contributes to monotonic increase in the dimension is the observation of new GM features. Rest of the state changes with each PoI but does not contribute to continuous increase of the overall dimensionality of the state vector.

Having defined the state vector and the cost function, Levenberg-Marquardt optimization could be performed by defining the derivatives with respect to the current state vector,

$$
\begin{aligned}
& \mathbf{A}=\mathbf{A}_{\mathbf{0}}+\mathbf{J}_{h}^{T} \mathbf{R}^{-1} \mathbf{J}_{h}+\mathbf{J}_{f}^{T} \mathbf{Q}^{-1} \mathbf{J}_{f} \\
& \mathbf{b}=\mathbf{b}_{0}+\mathbf{A}_{\mathbf{0}}\left(\hat{\mathbf{x}}_{0}-\hat{\mathbf{x}}^{-}\right)+\mathbf{J}_{h}^{T} \mathbf{R}^{-1} \boldsymbol{\varepsilon}_{x}+\mathbf{J}_{f}^{T} \mathbf{Q}^{-1} \boldsymbol{\varepsilon}_{y}
\end{aligned}
$$

Once optimization has converged LM features belonging to the current segment of the robot path are marginalized, corresponding observations, robot poses and any GM observations related to the removed robot poses are also removed. This smoothing cycle is applied recursively at each new PoI. Thus the consistency of the state estimate is preserved at regular intervals of the robots trajectory, while maintaining an efficient algorithm for arbitrary navigation.

\section{B. Marginalizing $L M$ from the $M M$}

The first requirement in the MMS approach is to remove the LM features from the state once they are used to improve the pose estimates of the relevant potion of the navigation. Consider the state vector in (11), which is the starting point for the Multi Map optimization at a given $\left(p^{\text {th }}\right) \mathrm{PoI}$,

$$
\mathbf{x}^{M M F}=\left[\mathbf{x}_{r, p}, \mathbf{x}_{m}^{M M F}\right]^{T}
$$

where $\mathbf{x}_{m}^{M M F}=\left[\mathbf{x}_{L M}, \mathbf{x}_{G M}\right]^{T}$ is the combined multi map at the current PoI. It is required to remove $\left(\mathbf{x}_{L M}\right)$ from $\left(\mathbf{x}_{m}^{M M F}\right)$. This could be achieved by straight forward removal of the corresponding map elements from the overall state vector. Thus,

$$
\mathbf{x}^{M M F}=\left[\mathbf{x}_{r, p}, \mathbf{x}_{G M}\right]^{T}
$$

However the optimization algorithm now contains a prior inverse covariance term which should also be marginalized accordingly. Assume that $\mathbf{A}_{0}$ is reordered to reflect the states to be removed $\left(\mathbf{A}_{L M, *}\right)$ and retained $\left(\mathbf{A}_{R, *}\right)$ such that,

$$
\mathbf{A}_{0}=\left[\begin{array}{cc}
\mathbf{A}_{L M, L M} & \mathbf{A}_{L M, R} \\
\mathbf{A}_{L M, R}^{T} & \mathbf{A}_{R, R}
\end{array}\right]
$$

Then using the matrix inversion lemma, the new prior after marginalization of LM could be calculated as,

$$
\mathbf{A}_{0} \leftarrow \mathbf{A}_{R, R}-\mathbf{A}_{L M, R}^{T} \mathbf{A}_{L M, L M}^{-1} \mathbf{A}_{L M, R}
$$

This technique lends itself to the removal of poses prior to the current PoI as well.

\section{Removing Observations Associated with Marginalized Features}

As the MM approach extends beyond the first loop closure it becomes necessary to remove observations corresponding to the previous LM features, since they are marginalized as described in the previous section. As a consequence the pose estimates belonging to these parts of the journey are no longer as accurate as would be when improved local navigation information was available through the LM. The technique described in section could be used to linearize such observations. The technique also lends itself to be used in linearizing any odometry measurements corresponding to marginalized robot poses in the state vector.

\section{RESULTS}

\section{A. Simulation Results}

Fig. 3 shows the map estimates from the MMS algorithm for a loop similar to that described in Fig 2. There are two nodes of the GM of which the first node initialized at the beginning of the robots trajectory aids the small loop closure and the second node first initialized during the third leg of the smaller loop aids the final loop closure. An EKF based SLAM was used to generate the initial estimate $\hat{\mathbf{x}}_{0}$. The estimated error from this filter is shown in Fig. 4(a) which as discussed in $[1,2]$ produce inconsistent state estimates. Fig. 4(b) shows the error estimate from the new MMS approach. As can be seen from the figure, the estimates are consistent and the two loop closures corresponding to the two nodes of the GM are also visible, indicated by the decreasing uncertainty.

Finally in Fig. 5, the average Normalized Estimation Error Squared (NEES) [14] for 50 trials of the simulation experiment are presented. The NEES is a measure of estimator consistency which could be used when the true locations of the states are known. The estimates are within the $2 \sigma$ confidence bounds conforming that the MMS is capable of producing consistent state estimates where EKF failed to produce consistent state estimates in comparably smaller sized loops using the particular stereo vision sensor as shown in $[1,2]$. 


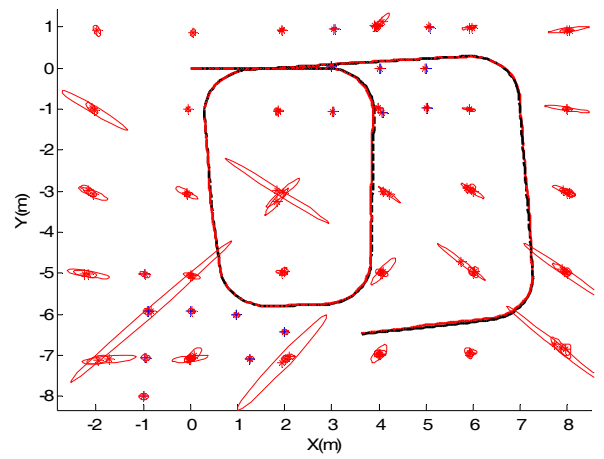

Fig. 3 Map estimate from the MMS. (a) The complete map comprising of the two nodes of GM ('+') and the LM ('*')
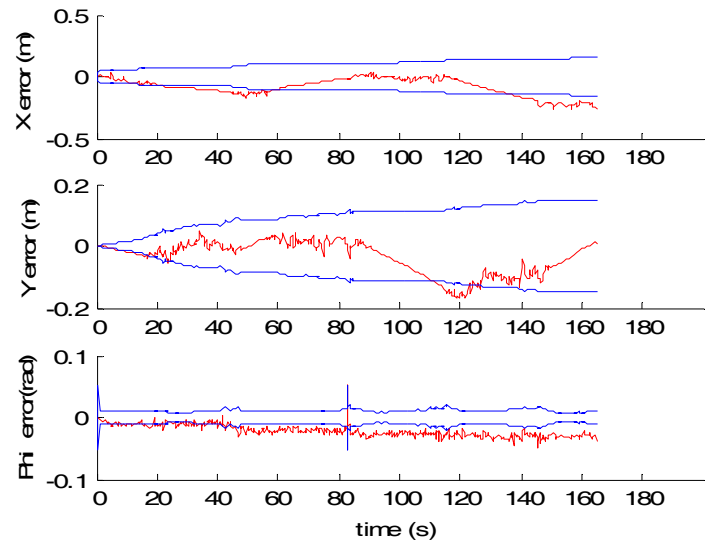

(a)
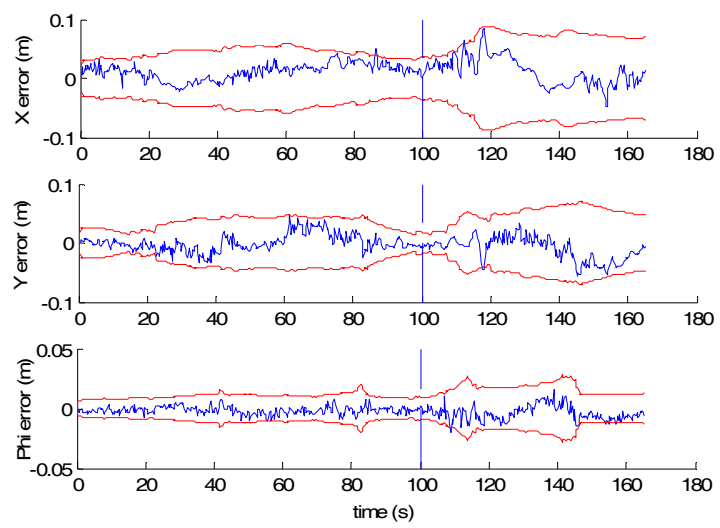

(b)

Fig. 4 Robot pose estimates from (a) EKF and (b) from the MM Smoothing. The first loop closure occurs near the $100 \mathrm{sec}$ corresponding to the first node of the GM

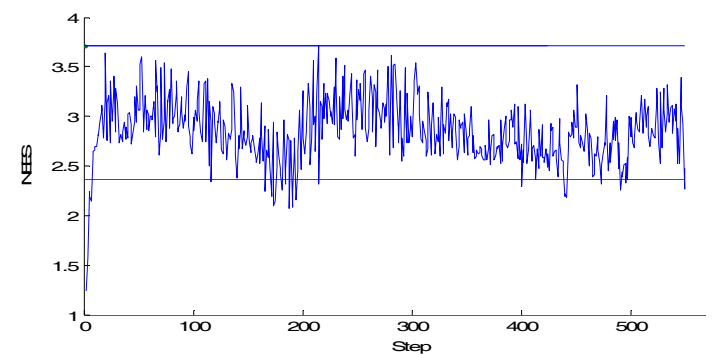

Fig. 5 Average NEES results for MMS

\section{B. Experimental results}

Below we asses the new framework in a practical indoor application using an Active Media Robotics Pioneer 2 as the robotic platform and videre stereo vision sensor. Fig. 6 shows a map of the environment generated using the pose estimates of the batch algorithm and raw laser scans. The thickness of the walls is a general indication of the error distribution in the robot path estimates. Therefore, quantitatively the algorithm provides sensible estimation of the robot path.

Fig. 7 shows the robot pose error relative to 'ground truth' given by a laser range finder based EKF. The outer (dashed) line is the compounded error estimate $\left(2 \sqrt{\sigma_{\text {laser }}^{2}+\sigma_{\text {vision }}^{2}}\right)$. The $2 \sigma$ error bounds from the batch algorithm as well as from the vision EKF are shown in the graph for comparison. As expected, vision EKF produced optimistic results. Apart from the error in $y$ estimate, both $x$ direction and heading errors appear to be consistently estimated relative to the compounded error bound. It is possible that the off shoot in y direction to be attributed to any accumulated errors in the laser EKF prior to the loop closure.

Fig. 8 shows the results of MMS applied to a large loop. For this experiment, SURF features were generated near the origin and near the end of the large loop enabling two loop closures, one after the completion of the smaller loop and the second after completion of the larger loop.

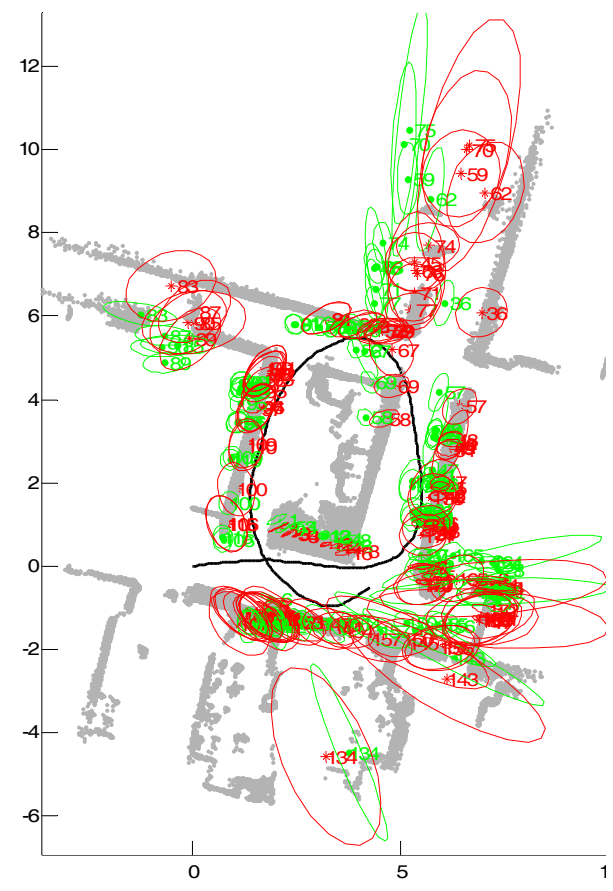

Fig. 6 SLAM results using the batch optimization algorithm. Red '*' indicates map estimates from the batch algorithm. Green '?' indicates the vision EKF map estimates. Green and red ellipses represent the covariance estimates from EKF and batch algorithms respectively. 

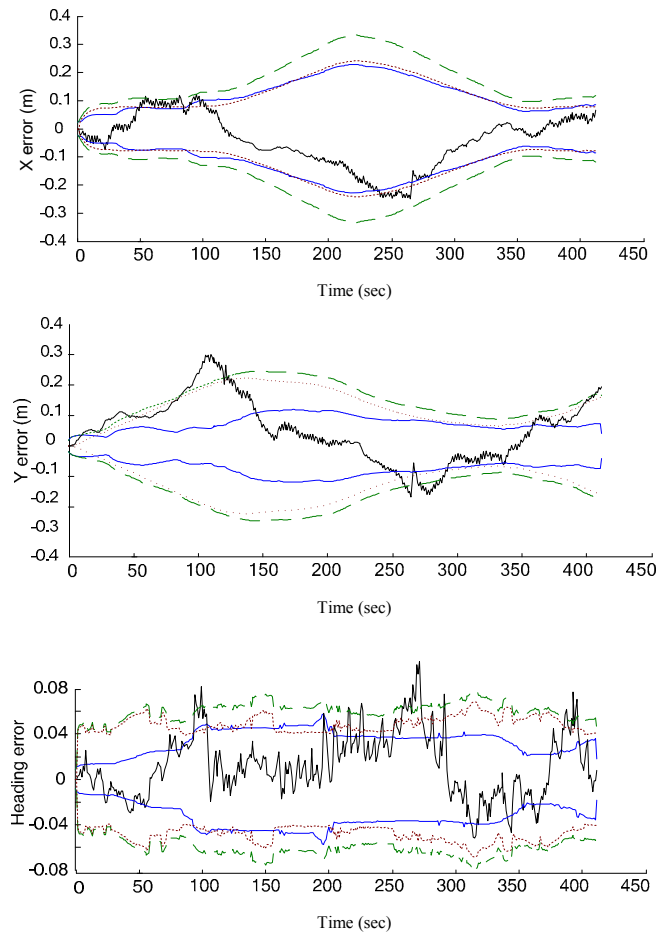

Fig. 7: Comparative robot $2 \sigma$ bounds (dashed lines indicate the combined estimate of laser and batch algorithm, dotted lines indicate estimates from the batch algorithm and the solid lines are from the vision EKF) and the error in Multi map SLAM robot pose estimation relative to the laser based EKF (ground truth)

\section{CONCLUSION}

This paper presented a novel framework for the small baseline stereo vision based SLAM The unique two tier map representation provides a compact method for representing large environments through a global map. The local map allows for temporary use of large number of features for local navigation. Marginalization of these features maintains the tractability of the developed algorithm within manageable constraints. With high dimensional feature descriptors, the global map provides the loop closure. We have shown that the multi map approach provided consistent results in challenging indoor environments. Further work is being carried out in order to generalize the framework as well as to study the computational aspects of the algorithm.

\section{REFERENCES}

D. C. Herath, K. R. S. Kodagoda, and G. Dissanayake, "Modeling Errors in Small Baseline Stereo for SLAM," in The 9 th International Conference on Control, Automation, Robotics and Vision (ICARCV 2006), Singapore, 2006.

[2] D. C. Herath, K. R. S. Kodagoda, and G. Dissanayake, "Stereo Vision Based SLAM: Issues and Solutions," in Vision Systems: Applications, G. Obinata and A. Dutta, Eds.: Pro literatur verlag, 2007, pp. 565-582.

[3] D. C. Herath, S. Kodagoda, and G. Dissanayake, "Simultaneous Localisation and Mapping: A Stereo Vision Based Approach," in IEEE/RSJ International Conference on Intelligent Robots and Systems (IROS 2006), Beijing, China, 2006, pp. 922-927.
Y. Bar-Shalom, X.-R. Li, and T. Kirubarajan, Estimation with Applications to Tracking and Navigation. Somerset, New Jersey: Wiley InterScience, 2001.

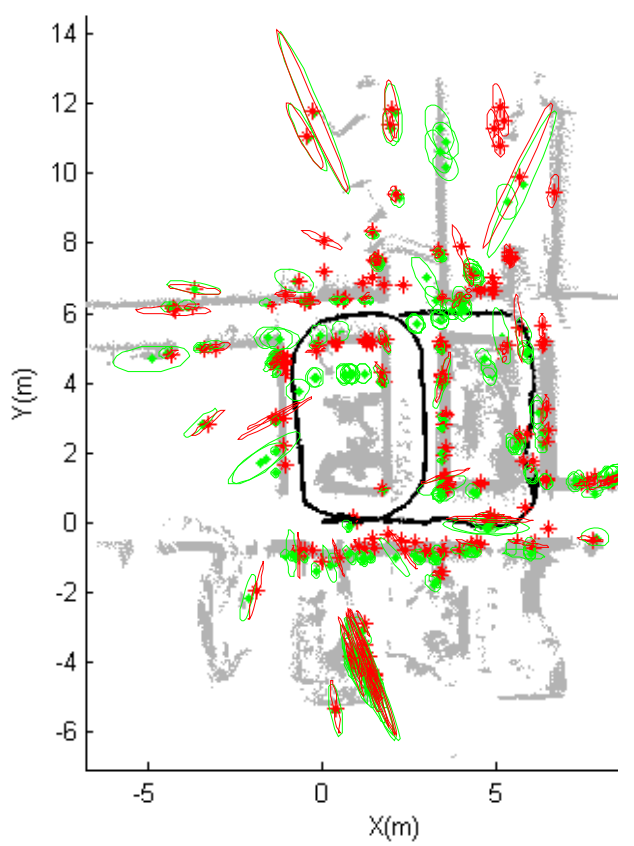

Fig. 8 Multi Map results of a large loop compromising two GM nodes. Red ' $*$ ' indicates the LM estimates given by the MMS and green ' ' corresponds to the same maps but the estimates are from the EKF, which provides the initial estimate for the MMS. 